\title{
Some fixed point theorems for contractive mappings of integral type
}

\author{
Zeqing Liu ${ }^{\mathrm{a}}$, Yuqing Wang ${ }^{\mathrm{a}}$, Shin Min Kang ${ }^{\mathrm{b}, \mathrm{c}}$, Young Chel Kwun ${ }^{\mathrm{d}, *}$ \\ ${ }^{a}$ Department of Mathematics, Liaoning Normal University, Dalian, Liaoning 116029, People's Republic of China. \\ ${ }^{b}$ Department of Mathematics and the RINS, Gyeongsang National University, Jinju 52828, Korea. \\ ${ }^{c}$ Center for General Education, China Medical University, Taichung 40402, Taiwan. \\ ${ }^{d}$ Department of Mathematics, Dong-A University, Busan 49315, Korea.
}

Communicated by S. S. Chang

\begin{abstract}
Four fixed point theorems for mappings satisfying contractive conditions of integral type in complete metric spaces are proved. The results presented in this paper extend and improve a few results existing in literature. Two examples involving the contractive mappings of integral type are constructed. (C)2017 All rights reserved.
\end{abstract}

Keywords: Contractive mappings of integral type, fixed point, complete metric space.

2010 MSC: $54 \mathrm{H} 25$.

\section{Introduction and preliminaries}

It is well-known that the Banach contraction principle is a very important result in the fixed point theory and has various generalizations and applications ([1-19]). In 2001, Rhoades [18] introduced the notion of $\varphi$-weakly contractive mappings and proved the following fixed point theorem, which generalizes the Banach contraction principle.

Theorem 1.1 ([18]). Let $\mathrm{f}$ be a mapping from a complete metric space $(\mathrm{X}, \mathrm{d})$ into itself satisfying

$$
d(f x, f y) \leqslant d(x, y)-\varphi(d(x, y)), \quad \forall x, y \in X,
$$

where

$$
\begin{aligned}
\varphi:[0,+\infty) & \rightarrow[0,+\infty) \text { is continuous and nondecreasing such that } \varphi \text { is positive on }(0,+\infty), \\
\varphi(0) & =0, \text { and } \lim _{\mathrm{t} \rightarrow+\infty} \varphi(\mathrm{t})=+\infty .
\end{aligned}
$$

Then $\mathrm{f}$ has a unique fixed point in $\mathrm{X}$.

\footnotetext{
${ }^{*}$ Corresponding author

Email addresses: zeqingliu@163.com (Zeqing Liu), yuqingwang93@163.com (Yuqing Wang), smkang@gnu.ac.kr (Shin Min Kang), yckwun@dau.ac.kr (Young Chel Kwun)
}

doi:10.22436/jnsa.010.07.17

Received 2017-03-30 
In 2008, Dutta and Choudhury [5] introduced the notion of $(\psi, \varphi)$-weakly contractive mappings and showed the following fixed point theorem, which extends Theorem 1.1.

Theorem 1.2 ([5]). Let $\mathrm{f}$ be a mapping from a complete metric space $(\mathrm{X}, \mathrm{d})$ into itself satisfying

$$
\psi(d(f x, f y)) \leqslant \psi(d(x, y))-\varphi(d(x, y)), \quad \forall x, y \in X
$$

where

$\psi:[0,+\infty) \rightarrow[0,+\infty)$ is continuous and nondecreasing function and $\varphi:[0,+\infty) \rightarrow[0,+\infty)$

is lower semicontinuous and nondecreasing function such that $\psi(t)=0=\varphi(t)$ if and only if $\mathrm{t}=0$.

Then $\mathrm{f}$ has a unique fixed point in $\mathrm{X}$.

In 2002, Branciari [3] gave an integral version of the Banach contraction principle and became the first to research on the existence of fixed points for contractive mappings of integral type.

Theorem 1.3 ([3]). Let $\mathrm{f}$ be a mapping from a complete metric space $(\mathrm{X}, \mathrm{d})$ into itself satisfying

$$
\int_{0}^{d(f x, f y)} \varphi(t) d t \leqslant c \int_{0}^{d(x, y)} \varphi(t) d t, \quad \forall x, y \in X,
$$

where

$$
\begin{aligned}
& \varphi:[0,+\infty) \rightarrow[0,+\infty) \text { is Lebesgue integrable which is summable on each compact } \\
& \text { subset of }[0,+\infty), \text { nonnegative, and such that for each } \varepsilon>0, \int_{0}^{\varepsilon} \varphi(t) d t>0
\end{aligned}
$$

Then $\mathrm{f}$ has a unique fixed point $\mathrm{a} \in \mathrm{X}$ such that $\lim _{\mathrm{n} \rightarrow \infty} \mathrm{f}^{\mathrm{n}} \mathrm{x}=\mathrm{a}$ for each $\mathrm{x} \in \mathrm{X}$.

Later on, the authors $[2,4,7-14,16,17]$ continued the study of Branciari and established a lot of fixed and common fixed point theorems for mappings satisfying contractive conditions of integral type. In particular, Rhoades [19] proved two fixed point theorems for mappings satisfying a general contractive inequality of integral type and generalized Theorem 1.3. Liu et al. [15] introduced some contractive mappings of integral type and proved the following fixed point results.

Theorem 1.4 ([15]). Let $\mathrm{f}$ be a mapping from a complete metric space $(\mathrm{X}, \mathrm{d})$ into itself satisfying

$$
\int_{0}^{d(f x, f y)} \varphi(t) d t \leqslant \int_{0}^{d(x, y)} \varphi(t) d t-\int_{0}^{\psi(d(x, y))} \varphi(t) d t, \quad \forall x, y \in X,
$$

where

$\varphi:[0,+\infty) \rightarrow[0,+\infty)$ is Lebesgue integrable, summable on each compact subset of $[0,+\infty)$

and $\int_{0}^{\varepsilon} \varphi(t) d t>0$ for each $\varepsilon>0, \psi:[0,+\infty) \rightarrow[0,+\infty)$ is lower semicontinuous function with $\psi(0)=0$ and $\psi(t)>0$ for each $\mathrm{t}>0$.

Then $\mathrm{f}$ has a unique fixed point $\mathrm{a} \in \mathrm{X}$ such that $\lim _{\mathrm{n} \rightarrow \infty} \mathrm{f}^{\mathrm{n}} \mathrm{x}=\mathrm{a}$ for each $\mathrm{x} \in \mathrm{X}$.

Theorem 1.5 ([15]). Let $\mathrm{f}$ be a mapping from a complete metric space $(X, d)$ into itself satisfying

$$
\int_{0}^{d(f x, f y)} \varphi(t) d t \leqslant \int_{0}^{M(x, y)} \varphi(t) d t-\int_{0}^{\psi(M(x, y))} \varphi(t) d t, \quad \forall x, y \in X,
$$

where

$$
M(x, y)=\max \left\{d(x, y), d(x, f x), d(y, f y), \frac{1}{2}[d(x, f y)+d(y, f x)]\right\}
$$

and $\varphi:[0,+\infty) \rightarrow[0,+\infty)$ is Lebesgue integrable, summable on each compact subset of $[0,+\infty)$ and $\int_{0}^{\varepsilon} \varphi(t) d t>$ 0 for each $\varepsilon>0, \psi:[0,+\infty) \rightarrow[0,+\infty)$ is lower semicontinuous function with $\psi(0)=0$ and $\psi(t)>0$ for each $t>0$. Then $\mathrm{f}$ has a unique fixed point $\mathrm{a} \in \mathrm{X}$ such that $\lim _{\mathrm{n} \rightarrow \infty} \mathrm{f}^{\mathrm{n}} \mathrm{x}=\mathrm{a}$ for each $\mathrm{x} \in \mathrm{X}$. 
The aim of this paper is to prove the existence, uniqueness and iterative approximations of fixed points for four classes of contractive mappings satisfying contractive conditions of integral type, which include the mappings (1.1), (1.3), (1.7), and (1.9) as special cases. Our results generalize Theorems 1.1, 1.2, 1.4, and 1.5. Two examples are constructed to show that our results differ from Theorems 1.1-1.4.

Throughout this paper, we assume that $\mathbb{R}^{+}=[0,+\infty), \mathbb{N}_{0}=\{0\} \cup \mathbb{N}$, where $\mathbb{N}$ denotes the set of all positive integers. Let $(X, d)$ be a metric space. For $f: X \rightarrow X$, define

$$
\begin{aligned}
x_{n+1}= & f x_{n}, \quad d_{n}=d\left(x_{n}, x_{n+1}\right), \quad \forall\left(n, x_{0}\right) \in \mathbb{N}_{0} \times X, \\
m_{1}(x, y)= & \max \left\{d(x, y), d(x, f x), d(y, f y), \frac{1}{2}[d(x, f y)+d(y, f x)], \frac{d(x, f x) d(y, f y)}{1+d(f x, f y)}, \frac{d(x, f y) d(y, f x)}{1+d(f x, f y)},\right. \\
& \left.\frac{d(x, f x) d(y, f y)}{1+d(x, y)}, \frac{d(x, f y) d(y, f x)}{1+d(x, y)}\right\}, \quad \forall x, y \in X, \\
m_{2}(x, y)= & \max \left\{d(x, y), d(x, f x), d(y, f y), \frac{1}{2}[d(x, f y)+d(y, f x)], \frac{d(x, f y)[1+d(y, f x)]}{2[1+d(x, y)]}, \frac{d(y, f x)[1+d(x, f y)]}{2[1+d(x, y)]},\right. \\
& \left.\frac{d(x, f x)[1+d(y, f y)]}{1+d(x, y)}, \frac{d(y, f y)[1+d(x, f x)]}{1+d(x, y)}\right\}, \quad \forall x, y \in X, \\
m_{3}(x, y)= & \max \left\{d(x, y), d(x, f x), d(y, f y), \frac{1}{2}[d(x, f y)+d(y, f x)]\right\}, \quad \forall x, y \in X .
\end{aligned}
$$

Let

- $\Phi_{1}=\left\{\varphi \mid \varphi: \mathbb{R}^{+} \rightarrow \mathbb{R}^{+}\right.$satisfies that $\varphi$ is Lebesgue integrable, summable on each compact subset of $\mathbb{R}^{+}$and $\int_{0}^{\varepsilon} \varphi(t) d t>0$ for each $\left.\varepsilon>0\right\}$;

- $\Phi_{2}=\left\{\varphi \mid \varphi: \mathbb{R}^{+} \rightarrow \mathbb{R}^{+}\right.$is nondecreasing continuous in $\mathbb{R}^{+} \backslash\{0\}$ and $\left.\varphi(t)=0 \Leftrightarrow t=0\right\}$;

- $\Phi_{3}=\left\{\varphi \mid \varphi: \mathbb{R}^{+} \rightarrow \mathbb{R}^{+}\right.$is a lower semicontinuous function and $\varphi(t)>0$ for each $\left.t>0\right\}$.

Lemma 1.6 ([11]). Let $\varphi \in \Phi_{1}$ and $\left\{r_{n}\right\}_{n \in \mathbb{N}}$ be a nonnegative sequence with $\lim _{n \rightarrow \infty} r_{n}=a$. Then

$$
\lim _{n \rightarrow \infty} \int_{0}^{r_{n}} \varphi(t) d t=\int_{0}^{a} \varphi(t) d t .
$$

\section{Several fixed point theorems}

In this section we show the existence, uniqueness and iterative approximations of fixed points for the contractive mappings (2.1), (2.14), (2.17), and (2.18).

Theorem 2.1. Let $\mathrm{f}$ be a mapping from a complete metric space $(\mathrm{X}, \mathrm{d})$ into itself satisfying

$$
\phi\left(\int_{0}^{\mathrm{d}(\mathrm{f} x, \mathrm{fy})} \varphi(\mathrm{t}) \mathrm{dt}\right) \leqslant \phi\left(\int_{0}^{\mathrm{m}_{1}(x, y)} \varphi(\mathrm{t}) \mathrm{dt}\right)-\int_{0}^{\psi\left(\mathrm{m}_{1}(x, y)\right)} \varphi(\mathrm{t}) \mathrm{dt}, \quad \forall x, y \in X,
$$

where $(\varphi, \phi, \psi) \in \Phi_{1} \times \Phi_{2} \times \Phi_{3}$. Then $\mathrm{f}$ has a unique fixed point $\mathrm{a} \in \mathrm{X}$ and $\lim _{\mathrm{n} \rightarrow \infty} \mathrm{f}^{\mathrm{n}} \mathrm{x}_{0}=\mathrm{a}$ for each $\mathrm{x}_{0} \in \mathrm{X}$.

Proof. Let $x_{0}$ be an arbitrary point in X. Suppose that there exists some $n_{0} \in \mathbb{N}_{0}$ with $x_{n_{0}}=x_{n_{0}+1}$. Clearly, $x_{n_{0}}=f x_{n_{0}}=\lim _{n \rightarrow \infty} f^{n} x_{n_{0}}$, that is, $x_{n_{0}}$ is a fixed point of $X$. Suppose that $x_{n} \neq x_{n+1}$ for each $n \in \mathbb{N}_{0}$. It follows that

$$
\begin{aligned}
m_{1}\left(x_{n-1}, x_{n}\right)=\max \{ & d\left(x_{n-1}, x_{n}\right), d\left(x_{n-1}, f x_{n-1}\right), d\left(x_{n}, f x_{n}\right), \frac{1}{2}\left[d\left(x_{n-1}, f x_{n}\right)+d\left(x_{n}, f x_{n-1}\right)\right], \\
& \frac{d\left(x_{n-1}, f x_{n-1}\right) d\left(x_{n}, f x_{n}\right)}{1+d\left(x_{n-1}, x_{n}\right)}, \frac{d\left(x_{n-1}, f x_{n}\right) d\left(x_{n}, f x_{n-1}\right)}{1+d\left(x_{n-1}, x_{n}\right)}, \\
& \left.\frac{d\left(x_{n-1}, f x_{n-1}\right) d\left(x_{n}, f x_{n}\right)}{1+d\left(f x_{n-1}, f x_{n}\right)}, \frac{d\left(x_{n-1}, f x_{n}\right) d\left(x_{n}, f x_{n-1}\right)}{1+d\left(f x_{n-1}, f x_{n}\right)}\right\}
\end{aligned}
$$




$$
\begin{aligned}
= & \max \left\{d\left(x_{n-1}, x_{n}\right), d\left(x_{n-1}, x_{n}\right), d\left(x_{n}, x_{n+1}\right), \frac{1}{2}\left[d\left(x_{n-1}, x_{n+1}\right)+d\left(x_{n}, x_{n}\right)\right],\right. \\
& \frac{d\left(x_{n-1}, x_{n}\right) d\left(x_{n}, x_{n+1}\right)}{1+d\left(x_{n-1}, x_{n}\right)}, \frac{d\left(x_{n-1}, x_{n+1}\right) d\left(x_{n}, x_{n}\right)}{1+d\left(x_{n-1}, x_{n}\right)}, \\
& \left.\frac{d\left(x_{n-1}, x_{n}\right) d\left(x_{n}, x_{n+1}\right)}{1+d\left(x_{n}, x_{n+1}\right)}, \frac{d\left(x_{n-1}, x_{n+1}\right) d\left(x_{n}, x_{n}\right)}{1+d\left(x_{n}, x_{n+1}\right)}\right\} \\
= & \max \left\{d_{n-1}, d_{n-1}, d_{n}, \frac{1}{2} d\left(x_{n-1}, x_{n+1}\right), \frac{d_{n-1} d_{n}}{1+d_{n-1}}, 0, \frac{d_{n-1} d_{n}}{1+d_{n}}, 0\right\} \\
= & \max \left\{d_{n-1}, d_{n}\right\}, \quad \forall n \in \mathbb{N} .
\end{aligned}
$$

Firstly, we show that

$$
\mathrm{d}_{\mathrm{n}} \leqslant \mathrm{d}_{\mathrm{n}-1,}, \quad \forall \mathrm{n} \in \mathbb{N}
$$

Suppose that (2.3) does not hold. It follows that there exists some $n_{0} \in \mathbb{N}$ satisfying

$$
\mathrm{d}_{\mathrm{n}_{0}}>\mathrm{d}_{\mathrm{n}_{0}-1} .
$$

Making use of (2.1), (2.2), (2.4), and $(\varphi, \phi, \psi) \in \Phi_{1} \times \Phi_{2} \times \Phi_{3}$, we conclude immediately that

$$
\begin{aligned}
0<\phi\left(\int_{0}^{d_{n_{0}}} \varphi(t) d t\right) & =\phi\left(\int_{0}^{d\left(x_{n_{0}}, x_{n_{0}+1}\right)} \varphi(t) d t\right) \\
& =\phi\left(\int_{0}^{d\left(f x_{n_{0}-1}, f x_{n_{0}}\right)} \varphi(t) d t\right) \\
& \leqslant \phi\left(\int_{0}^{m_{1}\left(x_{n_{0}-1}, x_{n_{0}}\right)} \varphi(t) d t\right)-\int_{0}^{\psi\left(m_{1}\left(x_{n_{0}-1}, x_{n_{0}}\right)\right)} \varphi(t) d t \\
& =\phi\left(\int_{0}^{\max \left\{d_{n_{0}-1}, d_{n_{0}}\right\}} \varphi(t) d t\right)-\int_{0}^{\psi\left(\max \left\{d_{n_{0}-1}, d_{n_{0}}\right\}\right)} \varphi(t) d t \\
& =\phi\left(\int_{0}^{d_{n_{0}}} \varphi(t) d t\right)-\int_{0}^{\psi\left(d_{n_{0}}\right)} \varphi(t) d t<\phi\left(\int_{0}^{d_{n_{0}}} \varphi(t) d t\right),
\end{aligned}
$$

which is a contradiction. Note that (2.3) means that there exists a constant $c$ with

$$
\lim _{n \rightarrow \infty} d_{n}=c \geqslant 0 .
$$

Secondly, we show that $c=0$. Suppose that $c>0$. Set $\liminf _{n \rightarrow \infty} \psi\left(d_{n}\right)=\alpha$. Obviously, there exists a subsequence $\left\{d_{n(k)-1}\right\}_{k \in \mathbb{N}}$ of $\left\{d_{n}\right\}_{n \in \mathbb{N}_{0}}$ such that $\lim _{k \rightarrow \infty} \psi\left(d_{n(k)-1}\right)=\alpha$. Since $\psi$ is lower semicontinuous, it follows from $\psi \in \Phi_{3}$ that $\alpha \geqslant \psi(c)>0$. On account of (2.1), (2.2), (2.3), (2.5), Lemma 1.6, and $(\varphi, \phi, \psi) \in \Phi_{1} \times \Phi_{2} \times \Phi_{3}$, we obtain that

$$
\begin{aligned}
& 0<\phi\left(\int_{0}^{c} \varphi(t) d t\right)=\limsup _{k \rightarrow \infty} \phi\left(\int_{0}^{d_{n(k)}} \varphi(t) d t\right) \\
& =\limsup _{k \rightarrow \infty} \phi\left(\int_{0}^{d\left(x_{n(k)}, x_{n(k)+1}\right)} \varphi(t) d t\right) \\
& =\limsup _{k \rightarrow \infty} \phi\left(\int_{0}^{d\left(f x_{n(k)-1}, f x_{n(k)}\right)} \varphi(t) d t\right) \\
& \leqslant \limsup _{k \rightarrow \infty}\left[\phi\left(\int_{0}^{m_{1}\left(x_{n(k)-1}, x_{n(k)}\right)} \varphi(t) d t\right)-\int_{0}^{\psi\left(m_{1}\left(x_{n(k)-1}, x_{n(k)}\right)\right)} \varphi(t) d t\right] \\
& =\limsup _{k \rightarrow \infty}\left[\phi\left(\int_{0}^{\max \left\{\mathrm{d}_{\mathfrak{n}(\mathrm{k})-1}, \mathrm{~d}_{\mathfrak{n}(\mathrm{k})}\right\}} \varphi(\mathrm{t}) \mathrm{dt}\right)-\int_{0}^{\psi\left(\max \left\{\mathrm{d}_{\mathfrak{n}(\mathrm{k})-1}, \mathrm{~d}_{\mathfrak{n}(\mathrm{k})}\right\}\right)} \varphi(\mathrm{t}) \mathrm{dt}\right]
\end{aligned}
$$




$$
\begin{aligned}
& =\limsup _{k \rightarrow \infty}\left[\phi\left(\int_{0}^{d_{n(k)-1}} \varphi(t) d t\right)-\int_{0}^{\psi\left(d_{n(k)-1}\right)} \varphi(t) d t\right] \\
& \leqslant \limsup _{k \rightarrow \infty} \phi\left(\int_{0}^{d_{n(k)-1}} \varphi(t) d t\right)-\liminf _{k \rightarrow \infty} \int_{0}^{\psi\left(d_{n(k)-1}\right)} \varphi(t) d t \\
& =\phi\left(\int_{0}^{c} \varphi(t) d t\right)-\int_{0}^{\alpha} \varphi(t) d t \\
& \leqslant \phi\left(\int_{0}^{c} \varphi(t) d t\right)-\int_{0}^{\psi(c)} \varphi(t) d t<\phi\left(\int_{0}^{c} \varphi(t) d t\right),
\end{aligned}
$$

which is a contradiction. Hence $c=0$ and

$$
\lim _{n \rightarrow \infty} d_{n}=0 .
$$

Thirdly, we show that $\left\{x_{n}\right\}_{\mathfrak{n} \in \mathbb{N}_{0}}$ is a Cauchy sequence. Suppose that $\left\{x_{\mathfrak{n}}\right\}_{\mathfrak{n} \in \mathbb{N}_{0}}$ is not a Cauchy sequence. It follows that there is a constant $\varepsilon>0$ and two subsequences $\left\{x_{m}(k)\right\}_{k \in \mathbb{N}}$ and $\left\{x_{n}(k)\right\}_{k \in \mathbb{N}}$ of $\left\{x_{\mathfrak{n}}\right\}_{\mathfrak{n} \in \mathbb{N}_{0}}$ such that $m(k)$ is minimal in the sense that $m(k)>n(k)>k$ and $d\left(x_{m(k)}, x_{n(k)}\right)>\varepsilon$. It follows that $d\left(x_{m(k)-1}, x_{n(k)}\right) \leqslant \varepsilon$. Observe that

$$
\begin{aligned}
\varepsilon & <d\left(x_{m(k)}, x_{n(k)}\right) \\
& \leqslant d\left(x_{m(k)}, x_{m(k)-1}\right)+d\left(x_{m(k)-1}, x_{n(k)-1}\right)+d\left(x_{n(k)-1}, x_{n(k)}\right) \\
& \leqslant d_{m(k)-1}+d\left(x_{m(k)-1}, x_{n(k)}\right)+d\left(x_{n(k)}, x_{n(k)-1}\right)+d_{n(k)-1} \\
& \leqslant d_{m(k)-1}+\varepsilon+2 d_{n(k)-1}, \quad \forall k \in \mathbb{N}
\end{aligned}
$$

and

$$
\begin{array}{ll}
\left|d\left(x_{m(k)-1}, x_{n(k)-1}\right)-d\left(x_{m(k)-1}, x_{n(k)}\right)\right| \leqslant d_{n(k)-1}, & \forall k \in \mathbb{N}, \\
\left|d\left(x_{m(k)-1}, x_{n(k)-1}\right)-d\left(x_{m(k)}, x_{n(k)-1}\right)\right| \leqslant d_{m(k)-1}, & \forall k \in \mathbb{N} .
\end{array}
$$

Letting $k \rightarrow \infty$ in (2.7), (2.8) and using (2.6), we infer that

$$
\begin{aligned}
\varepsilon=\lim _{k \rightarrow \infty} d\left(x_{m(k)}, x_{n(k)}\right) & =\lim _{k \rightarrow \infty} d\left(x_{m(k)-1}, x_{n(k)}\right) \\
& =\lim _{k \rightarrow \infty} d\left(x_{m(k)}, x_{n(k)-1}\right)=\lim _{k \rightarrow \infty} d\left(x_{m(k)-1}, x_{n(k)-1}\right) .
\end{aligned}
$$

On account of (2.6) and (2.9), we receive that

$$
\begin{aligned}
& \lim _{k \rightarrow \infty} m_{1}\left(x_{m}(k)-1, x_{n(k)-1}\right) \\
&=\lim _{k \rightarrow \infty} \max \left\{d\left(x_{m(k)-1}, x_{n(k)-1}\right), d\left(x_{m(k)-1}, f x_{m}(k)-1\right), d\left(x_{n(k)-1}, f x_{n(k)-1}\right),\right. \\
& \frac{1}{2}\left[d\left(x_{m(k)-1}, f x_{n(k)-1}\right)+d\left(x_{n(k)-1}, f x_{m(k)-1}\right)\right], \\
& \frac{d\left(x_{m(k)-1}, f x_{m(k)-1}\right) d\left(x_{n(k)-1}, f x_{n(k)-1}\right)}{1+d\left(x_{m(k)-1}, x_{n(k)-1}\right)}, \\
& \frac{d\left(x_{m(k)-1}, f x_{n(k)-1}\right) d\left(x_{n(k)-1}, f x_{m(k)-1}\right)}{1+d\left(x_{m}(k)-1, x_{n(k)-1}\right)}, \\
& \frac{d\left(x_{m(k)-1}, f x_{m(k)-1}\right) d\left(x_{n(k)-1}, f x_{n(k)-1}\right)}{1+d\left(f x_{m}(k)-1, f x_{n(k)-1}\right)}, \\
&\left.\frac{d\left(x_{m(k)-1}, f x_{n(k)-1}\right) d\left(x_{n(k)-1}, f x_{m(k)-1}\right)}{1+d\left(f x_{m}(k)-1, f x_{n(k)-1}\right)}\right\}
\end{aligned}
$$




$$
\begin{aligned}
= & \lim _{k \rightarrow \infty} \max \left\{d\left(x_{m(k)-1}, x_{n(k)-1}\right), d\left(x_{m(k)-1}, x_{m(k)}\right), d\left(x_{n(k)-1}, x_{n(k)}\right),\right. \\
& \frac{1}{2}\left[d\left(x_{m(k)-1}, x_{n(k)}\right)+d\left(x_{n(k)-1}, x_{m(k)}\right)\right], \\
& \frac{d\left(x_{m(k)-1}, x_{m(k)}\right) d\left(x_{n(k)-1}, x_{n(k)}\right)}{1+d\left(x_{m(k)-1}, x_{n(k)-1}\right)}, \frac{d\left(x_{m(k)-1}, x_{n(k)}\right) d\left(x_{n(k)-1}, x_{m(k)}\right)}{1+d\left(x_{m(k)-1}, x_{n(k)-1)}\right)} \\
& \left.\frac{d\left(x_{m(k)-1}, x_{m(k)}\right) d\left(x_{n(k)-1}, x_{n(k)}\right)}{1+d\left(x_{m}(k), x_{n(k)}\right)}, \frac{d\left(x_{m(k)-1}, x_{n(k)}\right) d\left(x_{n(k)-1}, x_{m(k)}\right)}{1+d\left(x_{m}(k), x_{n(k)}\right)}\right\} \\
= & \max \left\{\varepsilon, 0,0, \frac{1}{2}(\varepsilon+\varepsilon), 0, \frac{\varepsilon \cdot \varepsilon}{1+\varepsilon}, 0, \frac{\varepsilon \cdot \varepsilon}{1+\varepsilon}\right\} \\
= & \varepsilon .
\end{aligned}
$$

Put

$$
\liminf _{k \rightarrow \infty} \psi\left(m_{1}\left(x_{m}(k)-1, x_{n}(k)-1\right)\right)=\beta .
$$

Clearly, there exists a subsequence $\left\{m_{1}\left(x_{m\left(k_{j}\right)-1}, x_{n\left(k_{j}\right)-1}\right)\right\}_{j \in \mathbb{N}}$ of $\left\{d\left(x_{m(k)-1}, x_{n(k)-1}\right)\right\}_{k \in \mathbb{N}}$ such that

$$
\lim _{j \rightarrow \infty} \psi\left(m_{1}\left(x_{m}\left(k_{j}\right)-1, x_{n}\left(k_{j}\right)-1\right)\right)=\beta .
$$

Since $\psi$ is lower semicontinuous, it follows from (2.10)-(2.12) that $\beta \geqslant \psi(\varepsilon)>0$. In view of (2.1), (2.9)(2.12), Lemma 1.6, and $(\varphi, \phi, \psi) \in \Phi_{1} \times \Phi_{2} \times \Phi_{3}$, we deduce that

$$
\begin{aligned}
& 0<\phi\left(\int_{0}^{\varepsilon} \varphi(t) d t\right)=\limsup _{j \rightarrow \infty} \phi\left(\int_{0}^{d\left(x_{\mathfrak{m}\left(k_{j}\right), x_{n}\left(k_{j}\right)}\right)} \varphi(t) d t\right) \\
& =\limsup _{j \rightarrow \infty}\left[\phi\left(\int_{0}^{\mathrm{d}\left(\mathrm{f} x_{\mathfrak{m}\left(\mathrm{k}_{\mathbf{j}}\right)-1}, \mathrm{f} x_{\mathfrak{n}\left(\mathrm{k}_{\mathrm{j}}\right)-1}\right)} \varphi(\mathrm{t}) \mathrm{dt}\right)\right]
\end{aligned}
$$

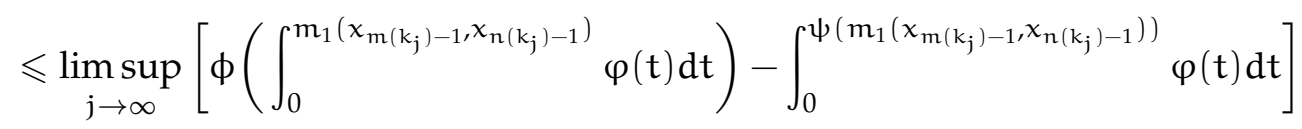

$$
\begin{aligned}
& \leqslant \limsup _{j \rightarrow \infty} \phi\left(\int_{0}^{m_{1}\left(x_{\mathfrak{m}\left(k_{j}\right)-1}, x_{n\left(k_{j}\right)-1}\right)} \varphi(t) d t\right)-\liminf _{j \rightarrow \infty} \int_{0}^{\psi\left(m_{1}\left(x_{m\left(k_{j}\right)-1}, x_{n\left(k_{j}\right)-1}\right)\right)} \varphi(t) d t \\
& =\phi\left(\int_{0}^{\varepsilon} \varphi(t) d t\right)-\int_{0}^{\beta} \varphi(t) d t \leqslant \phi\left(\int_{0}^{\varepsilon} \varphi(t) d t\right)-\int_{0}^{\psi(\varepsilon)} \varphi(t) d t<\phi\left(\int_{0}^{\varepsilon} \varphi(t) d t\right),
\end{aligned}
$$

which is a contradiction. Thus, $\left\{x_{n}\right\}_{n \in \mathbb{N}_{0}}$ is a Cauchy sequence. Since $(X, d)$ is a complete metric space, it follows that there exists a point $a \in X$ such that $\lim _{n \rightarrow \infty} x_{n}=a$.

Now we assert that $a=f a$. Suppose that $a \neq f a$. Note that

$$
\begin{aligned}
\lim _{n \rightarrow \infty} m_{1}\left(x_{n}, a\right)=\lim _{n \rightarrow \infty} \max \{ & d\left(x_{n}, a\right), d\left(x_{n}, f x_{n}\right), d(a, f a), \frac{1}{2}\left[d\left(x_{n}, f a\right)+d\left(a, f x_{n}\right)\right], \\
& \frac{d\left(x_{n}, f x_{n}\right) d(a, f a)}{1+d\left(x_{n}, a\right)}, \frac{d\left(x_{n}, f a\right) d\left(a, f x_{n}\right)}{1+d\left(x_{n}, a\right)}, \\
& \left.\frac{d\left(x_{n}, f x_{n}\right), d(a, f a)}{1+d\left(f x_{n}, f a\right)}, \frac{d\left(x_{n}, f a\right) d\left(a, f x_{n}\right)}{1+d\left(f x_{n}, f a\right)}\right\} \\
=\lim _{n \rightarrow \infty} \max \{ & d\left(x_{n}, a\right), d\left(x_{n}, x_{n+1}\right), d(a, f a), \frac{1}{2}\left[d\left(x_{n}, f a\right)+d\left(a, x_{n+1}\right)\right], \\
& \frac{d\left(x_{n}, x_{n+1}\right) d(a, f a)}{1+d\left(x_{n}, a\right)}, \frac{d\left(x_{n}, f a\right) d\left(a, x_{n+1}\right)}{1+d\left(x_{n}, a\right)},
\end{aligned}
$$




$$
\begin{array}{r}
\left.\frac{d\left(x_{n}, x_{n+1}\right) d(a, f a)}{1+d\left(x_{n+1}, f a\right)}, \frac{d\left(x_{n}, f a\right) d\left(a, x_{n+1}\right)}{1+d\left(x_{n+1}, f a\right)}\right\} \\
=\max \left\{0,0, d(a, f a), \frac{1}{2} d(a, f a), 0,0,0,0\right\}=d(a, f a) .
\end{array}
$$

Put

$$
\liminf _{n \rightarrow \infty} \psi\left(m_{1}\left(x_{n}, a\right)\right)=\gamma
$$

Clearly, there exists a subsequence $\left\{m_{1}\left(x_{n(j)}, a\right)\right\}_{j \in \mathbb{N}}$ of $\left\{m_{1}\left(x_{n}, a\right)\right\}_{n} \in \mathbb{N}$ such that

$$
\lim _{j \rightarrow \infty} \psi\left(m_{1}\left(x_{n(j)}, a\right)\right)=\gamma \geqslant \psi(d(a, f a))>0 .
$$

In view of (2.1), (2.13), Lemma 1.6, and $(\varphi, \phi, \psi) \in \Phi_{1} \times \Phi_{2} \times \Phi_{3}$, we deduce that

$$
\begin{aligned}
0<\phi\left(\int_{0}^{d(a, f a)} \varphi(t) d t\right) & =\limsup _{j \rightarrow \infty} \phi\left(\int_{0}^{d\left(x_{n(j)+1}, f a\right)} \varphi(t) d t\right) \\
& =\limsup _{j \rightarrow \infty} \phi\left(\int_{0}^{d\left(f x_{n(j)}, f a\right)} \varphi(t) d t\right) \\
& \leqslant \limsup _{j \rightarrow \infty}\left[\phi\left(\int_{0}^{m_{1}\left(x_{n(j)}, a\right)} \varphi(t) d t\right)-\int_{0}^{\psi\left(m_{1}\left(x_{n(j)}, a\right)\right)} \varphi(t) d t\right] \\
& \leqslant \limsup _{j \rightarrow \infty}\left(\int_{0}^{m_{1}\left(x_{n(j)}, a\right)} \varphi(t) d t\right)-\liminf _{j \rightarrow \infty} \int_{0}^{\psi\left(m_{1}\left(x_{n(j)}, a\right)\right)} \varphi(t) d t \\
& =\phi\left(\int_{0}^{d(a, f a)} \varphi(t) d t\right)-\int_{0}^{\gamma} \varphi(t) d t \\
& \leqslant \phi\left(\int_{0}^{d(a, f a)} \varphi(t) d t\right)-\int_{0}^{\psi(d(a, f a))} \varphi(t) d t<\phi\left(\int_{0}^{d(a, f a)} \varphi(t) d t\right),
\end{aligned}
$$

which is a contradiction. Thus, $a=f a$ is a fixed point of $f$ in $X$. Suppose that $f$ has another fixed point $\mathrm{b} \in \mathrm{X} \backslash\{\mathrm{a}\}$. Notice that

$$
\begin{aligned}
m_{1}(a, b)= & \max \left\{d(a, b), d(a, f a), d(b, f b), \frac{1}{2}[d(a, f b)+d(b, f a)], \frac{d(a, f a) d(b, f b)}{1+d(a, b)}, \frac{d(a, f b) d(b, f a)}{1+d(a, b)},\right. \\
& \left.\frac{d(a, f a) d(b, f b)}{1+d(f a, f b)}, \frac{d(a, f b) d(b, f a)}{1+d(f a, f b)}\right\} \\
= & \max \left\{d(a, b), 0,0, d(a, b), 0, \frac{d^{2}(a, b)}{1+d(a, b)}, 0, \frac{d^{2}(a, b)}{1+d(a, b)}\right\}=d(a, b),
\end{aligned}
$$

which together with (2.1), $\psi(\mathrm{d}(\mathrm{a}, \mathrm{b}))>0$, and $(\varphi, \phi, \psi) \in \Phi_{1} \times \Phi_{2} \times \Phi_{3}$ means that

$$
\begin{aligned}
\phi\left(\int_{0}^{\mathrm{d}(\mathrm{a}, \mathrm{b})} \varphi(\mathrm{t}) \mathrm{dt}\right) & =\phi\left(\int_{0}^{\mathrm{d}(\mathrm{fa}, \mathrm{fb})} \varphi(\mathrm{t}) \mathrm{dt}\right) \\
& \leqslant \phi\left(\int_{0}^{\mathrm{m}_{1}(\mathrm{a}, \mathrm{b})} \varphi(\mathrm{t}) \mathrm{dt}\right)-\int_{0}^{\psi\left(\mathrm{m}_{1}(\mathrm{a}, \mathrm{b})\right)} \varphi(\mathrm{t}) \mathrm{dt} \\
& =\phi\left(\int_{0}^{\mathrm{d}(\mathrm{a}, \mathrm{b})} \varphi(\mathrm{t}) \mathrm{dt}\right)-\int_{0}^{\psi(\mathrm{d}(\mathrm{a}, \mathrm{b}))} \varphi(\mathrm{t}) \mathrm{dt}<\phi\left(\int_{0}^{\mathrm{d}(\mathrm{a}, \mathrm{b})} \varphi(\mathrm{t}) \mathrm{dt}\right),
\end{aligned}
$$

which is a contradiction. This completes the proof. 
Theorem 2.2. Let $\mathrm{f}$ be a mapping from a complete metric space $(\mathrm{X}, \mathrm{d})$ into itself satisfying

$$
\phi\left(\int_{0}^{\mathrm{d}(\mathrm{f} x, \mathrm{fy})} \varphi(\mathrm{t}) \mathrm{dt}\right) \leqslant \phi\left(\int_{0}^{\mathrm{m}_{2}(x, y)} \varphi(\mathrm{t}) \mathrm{dt}\right)-\int_{0}^{\psi\left(\mathrm{m}_{2}(x, y)\right)} \varphi(\mathrm{t}) \mathrm{dt}, \quad \forall x, y \in X,
$$

where $(\varphi, \phi, \psi) \in \Phi_{1} \times \Phi_{2} \times \Phi_{3}$. Then $\mathrm{f}$ has a unique fixed point $\mathrm{a} \in \mathrm{X}$ and $\lim _{\mathrm{n} \rightarrow \infty} \mathrm{f}^{\mathrm{n}} \mathrm{x}_{0}=\mathrm{a}$ for each $\mathrm{x}_{0} \in \mathrm{X}$.

Proof. Let $x_{0}$ be an arbitrary point in $X$. Suppose that there exists some $n_{0} \in \mathbb{N}_{0}$ with $x_{n_{0}}=x_{n_{0}+1}$. Clearly, $x_{n_{0}}=f x_{n_{0}}=\lim _{n \rightarrow \infty} f^{n} x_{n_{0}}$, that is, $x_{n_{0}}$ is a fixed point of $f$. Suppose that $x_{n} \neq x_{n+1}$ for each $n \in \mathbb{N}_{0}$. Note that

$$
\left\{\frac{a+b}{2(1+a)}, \frac{a(1+b)}{1+a}\right\} \leqslant \max \{a, b\}, \quad \forall a, b \in \mathbb{R}^{+}
$$

and

$$
\begin{aligned}
m_{2}\left(x_{n-1}, x_{n}\right)=\max \left\{d\left(x_{n-1}, x_{n}\right), d\left(x_{n-1}, f x_{n-1}\right), d\left(x_{n}, f x_{n}\right), \frac{1}{2}\left[d\left(x_{n-1}, f x_{n}\right)+d\left(x_{n}, f x_{n-1}\right)\right],\right. & \frac{d\left(x_{n-1}, f x_{n-1}\right)\left[1+d\left(x_{n}, f x_{n}\right)\right]}{1+d\left(x_{n-1}, x_{n}\right)}, \frac{d\left(x_{n}, f x_{n}\right)\left[1+d\left(x_{n-1}, f x_{n-1}\right)\right]}{1+d\left(x_{n-1}, x_{n}\right)}, \\
& \left.\frac{d\left(x_{n-1}, f x_{n}\right)\left[1+d\left(x_{n}, f x_{n-1}\right)\right]}{2\left[1+d\left(x_{n-1}, x_{n}\right)\right]}, \frac{d\left(x_{n}, f x_{n-1}\right)\left[1+d\left(x_{n-1}, f x_{n}\right)\right]}{2\left[1+d\left(x_{n-1}, x_{n}\right)\right]}\right\} \\
= & \max \left\{d\left(x_{n-1}, x_{n}\right), d\left(x_{n-1}, x_{n}\right), d\left(x_{n}, x_{n+1}\right), \frac{1}{2}\left[d\left(x_{n-1}, x_{n+1}\right)+d\left(x_{n}, x_{n}\right)\right],\right. \\
& \frac{d\left(x_{n-1}, x_{n}\right)\left[1+d\left(x_{n}, x_{n+1}\right)\right]}{1+d\left(x_{n-1}, x_{n}\right)}, \frac{d\left(x_{n}, x_{n+1}\right)\left[1+d\left(x_{n-1}, x_{n}\right)\right]}{1+d\left(x_{n-1}, x_{n}\right)}, \\
& \left.\frac{d\left(x_{n-1}, x_{n+1}\right)\left[1+d\left(x_{n}, x_{n}\right)\right]}{2\left[1+d\left(x_{n-1}, x_{n}\right)\right]}, \frac{d\left(x_{n}, x_{n}\right)\left[1+d\left(x_{n-1}, x_{n+1}\right)\right]}{2\left[1+d\left(x_{n-1}, x_{n}\right)\right]}\right\} \\
= & \max \left\{d_{n-1}, d_{n-1}, d_{n}, \frac{1}{2} d\left(x_{n-1}, x_{n+1}\right), \frac{d_{n-1}\left(1+d_{n}\right)}{1+d_{n-1}}, d_{n}, \frac{d\left(x_{n-1}, x_{n+1}\right)}{2\left(1+d_{n-1}\right)}, 0\right\} \\
= & \max \left\{d_{n-1}, d_{n}\right\}, \quad \forall n \in \mathbb{N} .
\end{aligned}
$$

Firstly, we show that (2.3) holds. Suppose that (2.3) does not hold. It follows that there exists some $\mathrm{n}_{0} \in \mathbb{N}$ satisfying (2.4). Using (2.4), (2.14), (2.15), and $(\varphi, \phi, \psi) \in \Phi_{1} \times \Phi_{2} \times \Phi_{3}$, we obtain that

$$
\begin{aligned}
0<\phi\left(\int_{0}^{d_{n_{0}}} \varphi(t) d t\right) & =\phi\left(\int_{0}^{d\left(x_{n_{0}}, x_{n_{0}+1}\right)} \varphi(t) d t\right) \\
& =\phi\left(\int_{0}^{d\left(f x_{n_{0}-1}, f x_{n_{0}}\right)} \varphi(t) d t\right) \\
& \leqslant \phi\left(\int_{0}^{m_{2}\left(x_{n_{0}-1}, x_{n_{0}}\right)} \varphi(t) d t\right)-\int_{0}^{\psi\left(m_{2}\left(x_{n_{0}-1}, x_{n_{0}}\right)\right)} \varphi(t) d t \\
& =\phi\left(\int_{0}^{\max \left\{d_{n_{0}-1}, d_{n_{0}}\right\}} \varphi(t) d t\right)-\int_{0}^{\psi\left(\max \left\{d_{n_{0}-1}, d_{n_{0}}\right\}\right)} \varphi(t) d t \\
& =\phi\left(\int_{0}^{d_{n_{0}}} \varphi(t) d t\right)-\int_{0}^{\psi\left(d_{n_{0}}\right)} \varphi(t) d t<\phi\left(\int_{0}^{d_{n_{0}}} \varphi(t) d t\right),
\end{aligned}
$$

which is impossible and hence (2.3) holds. Note that (2.3) means that (2.5) holds.

Next, we show that $c=0$. Suppose that $c>0$. Set $\lim _{\inf } \ln _{n \rightarrow \infty} \psi\left(d_{n}\right)=\xi$. Obviously, there exists a subsequence $\left\{d_{n(k)}\right\}_{k \in \mathbb{N}}$ of $\left\{d_{n}\right\}_{n \in \mathbb{N}_{0}}$ such that $\lim _{k \rightarrow \infty} \psi\left(d_{n(k)-1}\right)=\xi$. Since $\psi$ is lower semicontinuous, it follows from $\psi \in \Phi_{3}$ that $\xi \geqslant \psi(c)>0$. On account of (2.5), (2.14), (2.15), Lemma 1.6, and $(\varphi, \phi, \psi) \in$ $\Phi_{1} \times \Phi_{2} \times \Phi_{3}$, we deduce that

$$
0<\phi\left(\int_{0}^{c} \varphi(t) d t\right)=\limsup _{k \rightarrow \infty} \phi\left(\int_{0}^{d_{n(k)}} \varphi(t) d t\right)
$$




$$
\begin{aligned}
& =\limsup _{k \rightarrow \infty} \phi\left(\int_{0}^{d\left(x_{n(k)}, x_{n(k)+1}\right)} \varphi(t) d t\right) \\
& =\underset{k \rightarrow \infty}{\limsup } \phi\left(\int_{0}^{d\left(f x_{n(k)-1}, f x_{n}(k)\right)} \varphi(t) d t\right) \\
& \leqslant \limsup _{k \rightarrow \infty}\left[\phi\left(\int_{0}^{m_{2}\left(x_{n(k)-1}, x_{n(k)}\right)} \varphi(t) d t\right)-\int_{0}^{\psi\left(m_{2}\left(x_{n(k)-1}, x_{n(k)}\right)\right)} \varphi(t) d t\right] \\
& =\limsup _{k \rightarrow \infty}\left[\phi\left(\int_{0}^{\max \left\{d_{n(k)-1}, d_{n(k)}\right\}} \varphi(t) d t\right)-\int_{0}^{\psi\left(\max \left\{d_{n(k)-1}, d_{n}(k)\right\}\right)} \varphi(t) d t\right] \\
& =\limsup _{k \rightarrow \infty}\left[\phi\left(\int_{0}^{d_{n(k)-1}} \varphi(t) d t\right)-\int_{0}^{\psi\left(d_{n(k)-1}\right)} \varphi(t) d t\right] \\
& \leqslant \limsup _{k \rightarrow \infty} \phi\left(\int_{0}^{d_{n(k)-1}} \varphi(t) d t\right)-\liminf _{k \rightarrow \infty}^{\psi} \int_{0}^{\psi\left(d_{n(k)-1}\right)} \varphi(t) d t \\
& =\phi\left(\int_{0}^{c} \varphi(t) d t\right)-\int_{0}^{\xi} \varphi(t) d t t \leqslant \phi\left(\int_{0}^{c} \varphi(t) d t\right)-\int_{0}^{\psi(c)} \varphi(t) d<\phi\left(\int_{0}^{c} \varphi(t) d t\right),
\end{aligned}
$$

which is a contradiction. Hence (2.6) holds.

Secondly, we prove that $\left\{x_{n}\right\}_{\mathfrak{n} \in \mathbb{N}_{0}}$ is a Cauchy sequence. Suppose that $\left\{x_{n}\right\}_{\mathfrak{n} \in \mathbb{N}_{0}}$ is not a Cauchy sequence. It follows that there is a constant $\varepsilon>0$ and two subsequences $\left\{x_{m(k)}\right\}_{k \in \mathbb{N}}$ and $\left\{x_{n(k)}\right\}_{k \in \mathbb{N}}$ of $\left\{x_{n}\right\}_{n \in \mathbb{N}_{0}}$ such that $m(k)$ is minimal in the sense that $m(k)>n(k)>k$ and $d\left(x_{m}(k), x_{n(k)}\right)>\varepsilon$. It follows that $d\left(x_{m(k)-1}, x_{n(k)}\right) \leqslant \varepsilon$, for all $k \in \mathbb{N}$. Similar to the proof of Theorem 2.1, we deduce (2.7)-(2.9). It follows that

$$
\begin{aligned}
& \lim _{k \rightarrow \infty} m_{2}\left(x_{m(k)-1}, x_{n(k)-1}\right) \\
& =\lim _{k \rightarrow \infty} \max \left\{d\left(x_{m(k)-1}, x_{n(k)-1}\right), d\left(x_{m(k)-1}, f x_{m(k)-1}\right), d\left(x_{n(k)-1}, f x_{n(k)-1}\right),\right. \\
& \frac{1}{2}\left[d\left(x_{m(k)-1}, f x_{n(k)-1}\right)+d\left(x_{n(k)-1}, f x_{m(k)-1}\right)\right], \\
& \frac{d\left(x_{m(k)-1}, f x_{m(k)-1}\right)\left[1+d\left(x_{n(k)-1}, f x_{n(k)-1}\right)\right]}{1+d\left(x_{m(k)-1}, x_{n(k)-1}\right)}, \\
& \frac{d\left(x_{n(k)-1}, f x_{n(k)-1}\right)\left[1+d\left(x_{m(k)-1}, f x_{m(k)-1}\right)\right]}{1+d\left(x_{m(k)-1}, x_{n(k)-1}\right)}, \\
& \frac{d\left(x_{m(k)-1}, f x_{n(k)-1}\right)\left[1+d\left(x_{n(k)-1}, f x_{m(k)-1}\right)\right]}{2\left[1+d\left(x_{m(k)-1}, x_{n(k)-1}\right)\right]}, \\
& \left.\frac{d\left(x_{n(k)-1}, f x_{m(k)-1}\right)\left[1+d\left(x_{m(k)-1}, f x_{n(k)-1}\right)\right]}{2\left[1+d\left(x_{m(k)-1}, x_{n(k)-1}\right)\right]}\right\} \\
& =\lim _{k \rightarrow \infty} \max \left\{d\left(x_{m}(k)-1, x_{n(k)-1}\right), d\left(x_{m(k)-1}, x_{m}(k)\right), d\left(x_{n}(k)-1, x_{n}(k)\right),\right. \\
& \frac{1}{2}\left[d\left(x_{m(k)-1}, x_{n(k)}\right)+d\left(x_{n(k)-1}, x_{m(k)}\right)\right], \\
& \frac{d\left(x_{m(k)-1}, x_{m(k)}\right)\left[1+d\left(x_{n(k)-1}, x_{n(k)}\right)\right]}{1+d\left(x_{m(k)-1}, x_{n(k)-1}\right)}, \frac{d\left(x_{n(k)-1}, x_{n(k)}\right)\left[1+d\left(x_{m(k)-1}, x_{m(k)}\right)\right]}{1+d\left(x_{m(k)-1}, x_{n(k)-1}\right)}, \\
& \left.\frac{d\left(x_{m(k)-1}, x_{n(k)}\right)\left[1+d\left(x_{n(k)-1}, x_{m(k)}\right)\right]}{2\left[1+d\left(x_{m(k)-1}, x_{n(k)-1}\right)\right]}, \frac{d\left(x_{n(k)-1}, x_{m(k)}\right)\left[1+d\left(x_{m(k)-1}, x_{n(k)}\right)\right]}{2\left[1+d\left(x_{m(k)-1}, x_{n(k)-1}\right)\right]}\right\} \\
& =\max \left\{\varepsilon, 0,0, \frac{1}{2}(\varepsilon+\varepsilon), 0,0, \frac{\varepsilon(1+\varepsilon)}{2(1+\varepsilon)}, \frac{\varepsilon(1+\varepsilon)}{2(1+\varepsilon)}\right\}=\varepsilon \text {. }
\end{aligned}
$$


It is clear that (2.11) and (2.12) hold. It follows from (2.11) and (2.12) that $\beta \geqslant \psi(\varepsilon)>0$. In light of (2.14), (2.16), Lemma 1.6, and $(\varphi, \phi, \psi) \in \Phi_{1} \times \Phi_{2} \times \Phi_{3}$, we deduce that

$$
\begin{aligned}
& 0<\phi\left(\int_{0}^{\varepsilon} \varphi(t) d t\right)=\limsup _{j \rightarrow \infty} \phi\left(\int_{0}^{d\left(x_{m\left(k_{j}\right)}, x_{n\left(k_{j}\right)}\right)} \varphi(t) d t\right)
\end{aligned}
$$

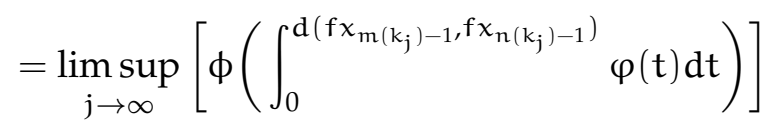

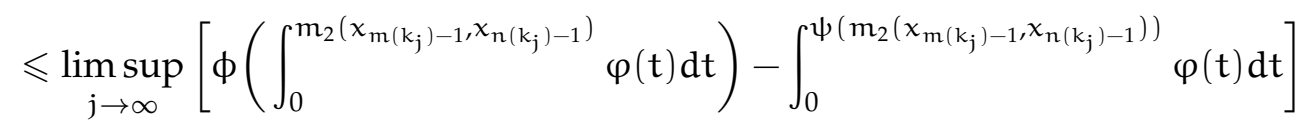

$$
\begin{aligned}
& \leqslant \limsup _{j \rightarrow \infty} \phi\left(\int_{0}^{m_{2}\left(x_{m}\left(k_{j}\right)-1, x_{n\left(k_{j}\right)-1}\right)} \varphi(t) d t\right)-\liminf _{n \rightarrow \infty} \int_{0}^{\psi\left(m_{2}\left(x_{m\left(k_{j}\right)-1}, x_{n\left(k_{j}\right)-1}\right)\right)} \varphi(t) d t \\
& =\phi\left(\int_{0}^{\varepsilon} \varphi(t) d t\right)-\int_{0}^{\beta} \varphi(t) d t \leqslant \phi\left(\int_{0}^{\varepsilon} \varphi(t) d t\right)-\int_{0}^{\psi(\varepsilon)} \varphi(t) d t<\phi\left(\int_{0}^{\varepsilon} \varphi(t) d t\right),
\end{aligned}
$$

which is a contradiction. Thus, $\left\{x_{n}\right\}_{n \in \mathbb{N}_{0}}$ is a Cauchy sequence. Since $(X, d)$ is a complete metric space, it follows that there exists a point $a \in X$ such that $\lim _{n \rightarrow \infty} x_{n}=a$.

Thirdly, we assert that $a=f a$. Suppose that $a \neq f a$. Note that (2.6) and $\lim _{n \rightarrow \infty} x_{n}=a$ yield that

$$
\begin{aligned}
\lim _{n \rightarrow \infty} m_{2}\left(x_{n}, a\right)=\lim _{n \rightarrow \infty} \max \left\{d\left(x_{n}, a\right), d\left(x_{n}, f x_{n}\right), d(a, f a), \frac{1}{2}\left[d\left(x_{n}, f a\right)+d\left(a, f x_{n}\right)\right],\right. & \\
& \frac{d\left(x_{n}, f x_{n}\right)[1+d(a, f a)]}{1+d\left(x_{n}, a\right)}, \frac{d(a, f a)\left[1+d\left(x_{n}, f x_{n}\right)\right]}{1+d\left(x_{n}, a\right)}, \\
& \left.\frac{d\left(x_{n}, f a\right)\left[1+d\left(a, f x_{n}\right)\right]}{2\left[1+d\left(x_{n}, a\right)\right]}, \frac{d\left(a, f x_{n}\right)\left[1+d\left(x_{n}, f a\right)\right]}{2\left[1+d\left(x_{n}, a\right)\right]}\right\} \\
=\lim _{n \rightarrow \infty} \max \left\{d\left(x_{n}, a\right), d\left(x_{n}, x_{n+1}\right), d(a, f a), \frac{1}{2}\left[d\left(x_{n}, f a\right)+d\left(a, x_{n+1}\right)\right],\right. & \frac{d\left(x_{n}, x_{n+1}\right)[1+d(a, f a)]}{1+d\left(x_{n}, a\right)}, \frac{d(a, f a)\left[1+d\left(x_{n}, x_{n+1}\right)\right]}{1+d\left(x_{n}, a\right)}, \\
& \left.\frac{d\left(x_{n}, f a\right)\left[1+d\left(a, x_{n+1}\right)\right]}{2\left[1+d\left(x_{n}, a\right)\right]}, \frac{d\left(a, x_{n+1}\right)\left[1+d\left(x_{n}, f a\right)\right]}{2\left[1+d\left(x_{n}, a\right)\right]}\right\} \\
= & \max \left\{0,0, d(a, f a), \frac{1}{2} d(a, f a), 0, d(a, f a), \frac{d(a, f a)}{2}, 0\right\}=d(a, f a) .
\end{aligned}
$$

Put

$$
\liminf _{n \rightarrow \infty} \psi\left(m_{2}\left(a, x_{n}\right)\right)=\eta .
$$

Clearly, there exists a subsequence $\left\{m_{2}\left(a, x_{n}(j)\right)\right\}_{j \in \mathbb{N}}$ of $\left\{m_{2}\left(a, x_{n}\right)\right\}_{\mathfrak{n} \in \mathbb{N}}$ such that

$$
\lim _{j \rightarrow \infty} \psi\left(m_{2}\left(a, x_{n(j)}\right)\right)=\eta \geqslant \psi(d(a, f a)) .
$$

In view of (2.14), Lemma 1.6, and $(\varphi, \phi, \psi) \in \Phi_{1} \times \Phi_{2} \times \Phi_{3}$, we give that

$$
\begin{aligned}
0<\phi\left(\int_{0}^{d(a, f a)} \varphi(t) d t\right) & =\limsup _{j \rightarrow \infty} \phi\left(\int_{0}^{d\left(x_{n(j)+1}, f a\right)} \varphi(t) d t\right) \\
& =\limsup _{j \rightarrow \infty} \phi\left(\int_{0}^{d\left(f x_{n(j)}, f a\right)} \varphi(t) d t\right) \\
& \leqslant \limsup _{j \rightarrow \infty}\left[\phi\left(\int_{0}^{m_{2}\left(x_{n(j)}, a\right)} \varphi(t) d t\right)-\int_{0}^{\psi\left(m_{2}\left(x_{n(j)}, a\right)\right)} \varphi(t) d t\right]
\end{aligned}
$$




$$
\begin{aligned}
& \leqslant \underset{j \rightarrow \infty}{\limsup } \phi\left(\int_{0}^{m_{2}\left(x_{n(j)}, a\right)} \varphi(t) d t\right)-\liminf _{j \rightarrow \infty} \int_{0}^{\psi\left(m_{2}\left(x_{n(j)}, a\right)\right)} \varphi(t) d t \\
& =\phi\left(\int_{0}^{d(a, f a)} \varphi(t) d t\right)-\int_{0}^{\eta} \varphi(t) d t \\
& \leqslant \phi\left(\int_{0}^{d(a, f a)} \varphi(t) d t\right)-\int_{0}^{\psi(d(a, f a))} \varphi(t) d t<\phi\left(\int_{0}^{d(a, f a)} \varphi(t) d t\right),
\end{aligned}
$$

which is impossible. Thus, $a=f a$. Suppose that $f$ has another fixed point $b \in X \backslash\{a\}$. Note that

$$
\begin{aligned}
& m_{2}(a, b)=\max \left\{d(a, b), d(a, f a), d(b, f b), \frac{1}{2}[d(a, f b)+d(b, f a)],\right. \\
& \frac{d(a, f a)[1+d(b, f b)]}{1+d(a, b)} \frac{d(b, f b)[1+d(a, f a)]}{1+d(a, b)}, \\
&\left.\frac{d(a, f b)[1+d(b, f a)]}{2[1+d(a, b)]}, \frac{d(b, f a)[1+d(a, f b)]}{2[1+d(a, b)]}\right\} \\
&= \max \left\{d(a, b), 0,0, d(a, b), 0,0, \frac{d(a, b)}{2}, \frac{d(a, b)}{2}\right\}=d(a, b),
\end{aligned}
$$

which together with (2.14), $\psi(d(a, b))>0$, and $(\varphi, \phi, \psi) \in \Phi_{1} \times \Phi_{2} \times \Phi_{3}$ means that

$$
\begin{aligned}
\phi\left(\int_{0}^{\mathrm{d}(\mathrm{a}, \mathrm{b})} \varphi(\mathrm{t}) \mathrm{dt}\right) & =\phi\left(\int_{0}^{\mathrm{d}(\mathrm{fa}, \mathrm{fb})} \varphi(\mathrm{t}) \mathrm{dt}\right) \\
& \leqslant \phi\left(\int_{0}^{\mathrm{m}_{2}(\mathrm{a}, \mathrm{b})} \varphi(\mathrm{t}) \mathrm{dt}\right)-\int_{0}^{\psi\left(\mathrm{m}_{2}(\mathrm{a}, \mathrm{b})\right)} \varphi(\mathrm{t}) \mathrm{dt} \\
& =\phi\left(\int_{0}^{\mathrm{d}(\mathrm{a}, \mathrm{b})} \varphi(\mathrm{t}) \mathrm{dt}\right)-\int_{0}^{\psi(d(a, b))} \varphi(\mathrm{t}) \mathrm{dt}<\phi\left(\int_{0}^{\mathrm{d}(\mathrm{a}, \mathrm{b})} \varphi(\mathrm{t}) \mathrm{dt}\right),
\end{aligned}
$$

which is impossible. This completes the proof.

Similar to the proofs of Theorems 2.1 and 2.2, we have the following results.

Theorem 2.3. Let $\mathrm{f}$ be a mapping from a complete metric space $(\mathrm{X}, \mathrm{d})$ into itself satisfying

$$
\phi\left(\int_{0}^{\mathrm{d}(\mathrm{f} x, \mathrm{fy})} \varphi(\mathrm{t}) \mathrm{dt}\right) \leqslant \phi\left(\int_{0}^{\mathrm{m}_{3}(x, y)} \varphi(\mathrm{t}) \mathrm{dt}\right)-\int_{0}^{\psi\left(\mathrm{m}_{3}(x, y)\right)} \varphi(\mathrm{t}) \mathrm{dt}, \quad \forall x, y \in X,
$$

where $(\varphi, \phi, \psi) \in \Phi_{1} \times \Phi_{2} \times \Phi_{3}$. Then $\mathrm{f}$ has a unique fixed point $\mathrm{a} \in \mathrm{X}$ and $\lim _{\mathrm{n} \rightarrow \infty} \mathrm{f}^{\mathrm{n}} \mathrm{x}_{0}=\mathrm{a}$ for each $\mathrm{x}_{0} \in \mathrm{X}$.

Theorem 2.4. Let $\mathrm{f}$ be a mapping from a complete metric space $(\mathrm{X}, \mathrm{d})$ into itself satisfying

$$
\phi\left(\int_{0}^{\mathrm{d}(f x, f y)} \varphi(t) d t\right) \leqslant \phi\left(\int_{0}^{\mathrm{d}(x, y)} \varphi(\mathrm{t}) \mathrm{dt}\right)-\int_{0}^{\psi(\mathrm{d}(\mathrm{x}, \mathrm{y}))} \varphi(\mathrm{t}) \mathrm{dt}, \quad \forall x, y \in X,
$$

where $(\varphi, \phi, \psi) \in \Phi_{1} \times \Phi_{2} \times \Phi_{3}$. Then $\mathrm{f}$ has a unique fixed point $\mathrm{a} \in \mathrm{X}$ and $\lim _{\mathrm{n} \rightarrow \infty} \mathrm{f}^{\mathrm{n}} \mathrm{x}_{0}=\mathrm{a}$ for each $\mathrm{x}_{0} \in \mathrm{X}$.

Remark 2.5. It is clear that Theorem 2.3 extends Theorem 1.5, and Theorem 2.4 generalizes Theorems 1.1, 1.2, and 1.4. The examples below are applications of Theorems 2.1-2.4 and show that Theorems 2.1-2.3 differ from Theorems 1.1-1.4.

Example 2.6. Let $X=\mathbb{R}^{+}$be endowed with Euclidean metric $d=|\cdot|, f: X \rightarrow X$ and $\varphi, \phi, \psi: \mathbb{R}^{+} \rightarrow \mathbb{R}^{+}$ be defined by

$$
f x= \begin{cases}1, & \forall x \in \mathbb{R}^{+} \backslash\left\{\frac{1}{8}\right\} \\ \frac{7}{8}, & x=\frac{1}{8}\end{cases}
$$


and

$$
\varphi(t)=4 \mathrm{t}^{3}, \quad \phi(\mathrm{t})=\mathrm{t}, \quad \forall \mathrm{t} \in \mathbb{R}^{+}, \quad \psi(\mathrm{t})= \begin{cases}\frac{3}{4} \mathrm{t}, & \forall \mathrm{t} \in\left[0, \frac{3}{4}\right), \\ \frac{1}{4}, & \forall \mathrm{t} \in\left[\frac{3}{4},+\infty\right) .\end{cases}
$$

Clearly, $(X, d)$ is a complete metric space and $(\varphi, \phi, \psi) \in \Phi_{1} \times \Phi_{2} \times \Phi_{3}$. Let $x, y \in X$. In order to verify (2.1), (2.14), and (2.17), we have to consider the following two possible cases.

Case 1. $x, y \in \mathbb{R}^{+} \backslash\left\{\frac{1}{8}\right\}$ or $x=y=\frac{1}{8}$. Observe that $\psi(t) \leqslant t$, for all $t \in \mathbb{R}^{+}$. Obviously,

$$
\begin{aligned}
\phi\left(\int_{0}^{d(f x, f y)} \varphi(t) d t\right)=\phi(0)=0 & \leqslant\left(m_{i}(x, y)\right)^{4}-\left(\psi\left(m_{i}(x, y)\right)\right)^{4} \\
& =\phi\left(\int_{0}^{m_{i}(x, y)} \varphi(t) d t\right)-\int_{0}^{\psi\left(m_{i}(x, y)\right)} \varphi(t) d t, \quad i \in\{1,2,3\}
\end{aligned}
$$

Case 2. $x \in \mathbb{R}^{+} \backslash\left\{\frac{1}{8}\right\}$ and $y=\frac{1}{8}$ or $x=\frac{1}{8}$ and $y \in \mathbb{R}^{+} \backslash\left\{\frac{1}{8}\right\}$. Note that $\phi$ is nondecreasing in $\mathbb{R}^{+}$. It follows that

$$
m_{i}(x, y) \geqslant \max \{|x-f x|,|y-f y|\} \geqslant \frac{3}{4}, \quad i \in\{1,2,3\},
$$

and

$$
\begin{aligned}
\phi\left(\int_{0}^{\mathrm{d}(\mathrm{fx}, \mathrm{fy})} \varphi(\mathrm{t}) \mathrm{dt}\right)=\phi\left(\frac{1}{8^{4}}\right)=\frac{1}{4096} & <\frac{81}{256}-\frac{1}{256} \\
& =\phi\left(\left(\frac{3}{4}\right)^{4}\right)-\left(\psi\left(\frac{3}{4}\right)\right)^{4} \\
& =\phi\left(\int_{0}^{\frac{3}{4}} \varphi(\mathrm{t}) \mathrm{dt}\right)-\int_{0}^{\psi\left(\frac{3}{4}\right)} \varphi(\mathrm{t}) \mathrm{dt} \\
& \leqslant \phi\left(\int_{0}^{\mathrm{m}_{\mathfrak{i}}(\mathrm{x}, \mathrm{y})} \varphi(\mathrm{t}) \mathrm{dt}\right)-\int_{0}^{\psi\left(\mathrm{m}_{\mathrm{i}}(x, y)\right)} \varphi(\mathrm{t}) \mathrm{dt}, \quad i \in\{1,2,3\} .
\end{aligned}
$$

That is, (2.1), (2.14), and (2.17) hold. Thus each of Theorems 2.1-2.3 guarantees that $f$ has a unique fixed point in $X$.

However, Theorems 1.1-1.4 are useless in proving the existence of fixed points of $f$ in $X$.

Suppose that $f$ satisfies the conditions of Theorem 1.1. That is, there exists $\varphi: \mathbb{R}^{+} \rightarrow \mathbb{R}^{+}$satisfying (1.1) and (1.2). It follows from (1.1) and (1.2) that

$$
\frac{1}{8}=d\left(f \frac{1}{16}, f \frac{1}{8}\right) \leqslant d\left(\frac{1}{16}, \frac{1}{8}\right)-\varphi\left(d\left(\frac{1}{16}, \frac{1}{8}\right)\right)=\frac{1}{16}-\varphi\left(\frac{1}{16}\right)<\frac{1}{16},
$$

which is a contradiction.

Suppose that $f$ satisfies the conditions of Theorem 1.2. That is, there exist $\psi, \varphi: \mathbb{R}^{+} \rightarrow \mathbb{R}^{+}$satisfying (1.3) and (1.4). Using (1.3) and (1.4), we infer that

$$
\psi\left(\frac{1}{8}\right)=\psi\left(\mathrm{d}\left(\mathrm{f} \frac{1}{32}, \mathrm{f} \frac{1}{8}\right)\right) \leqslant \psi\left(\mathrm{d}\left(\frac{1}{32}, \frac{1}{8}\right)\right)-\varphi\left(\mathrm{d}\left(\frac{1}{32}, \frac{1}{8}\right)\right)=\psi\left(\frac{3}{32}\right)-\varphi\left(\frac{3}{32}\right),
$$

that is,

$$
0<\varphi\left(\frac{3}{32}\right) \leqslant \psi\left(\frac{3}{32}\right)-\psi\left(\frac{1}{8}\right) \leqslant 0
$$

which is impossible.

Suppose that $f$ satisfies the conditions of Theorem 1.3. That is, there exists $\varphi: \mathbb{R}^{+} \rightarrow \mathbb{R}^{+}$satisfying (1.5) and (1.6). In view of (1.5) and (1.6), we conclude that

$$
0<\int_{0}^{\frac{1}{8}} \varphi(t) d t=\int_{0}^{d\left(1, \frac{7}{8}\right)} \varphi(t) d t=\lim _{x \rightarrow \frac{1}{8}} \int_{0}^{d\left(f x, f \frac{1}{8}\right)} \varphi(t) d t \leqslant \lim _{x \rightarrow \frac{1}{8}} c \int_{0}^{d\left(x, \frac{1}{8}\right)} \varphi(t) d t=0,
$$


which is a contradiction.

Suppose that $f$ satisfies the conditions of Theorem 1.4 . That is, there exist $\psi, \varphi: \mathbb{R}^{+} \rightarrow \mathbb{R}^{+}$satisfying (1.7) and (1.8). In light of (1.7) and (1.8), we deduce that

$$
\begin{aligned}
0<\int_{0}^{\frac{1}{8}} \varphi(t) d t=\int_{0}^{d\left(\frac{7}{8}, 1\right)} \varphi(t) d t & =\lim _{y \rightarrow \frac{1}{8}} \int_{0}^{d\left(f \frac{1}{8}, f y\right)} \varphi(t) d t \\
& \leqslant \lim _{y \rightarrow \frac{1}{8}}\left(\int_{0}^{d\left(\frac{1}{8}, y\right)} \varphi(t) d t-\int_{0}^{\psi\left(d\left(\frac{1}{8}, y\right)\right)} \varphi(t) d t\right) \leqslant \lim _{y \rightarrow \frac{1}{8}} \int_{0}^{d\left(\frac{1}{8}, y\right)} \varphi(t) d t=0,
\end{aligned}
$$

which is absurd.

Example 2.7. Let $X=\left[0, \frac{1}{2}\right] \cup\{1,3\}$ be endowed with Euclidean metric $d=|\cdot|, f: X \rightarrow X$ and $\varphi, \phi, \psi:$ $\mathbb{R}^{+} \rightarrow \mathbb{R}^{+}$be defined by

$$
f x=\left\{\begin{array}{ll}
\frac{x}{2}, & \forall x \in\left[0, \frac{1}{2}\right], \\
0, & x=1, \\
1, & x=3,
\end{array} \quad \varphi(t)= \begin{cases}2 t, & \forall t \in[0,1] \\
1, & \forall t \in(1,+\infty)\end{cases}\right.
$$

and

$$
\phi(t)=\left\{\begin{array}{ll}
\mathrm{t}, & \forall \mathrm{t} \in[0,1], \\
\frac{\mathrm{t}+1}{2}, & \forall \mathrm{t} \in(1,+\infty),
\end{array} \quad \psi(\mathrm{t})= \begin{cases}\frac{\mathrm{t}}{2}, & \forall \mathrm{t} \in[0,1), \\
\frac{1}{4}, & \forall \mathrm{t} \in[1,+\infty) .\end{cases}\right.
$$

Obviously, $(X, d)$ is a complete metric space and $(\varphi, \phi, \psi) \in \Phi_{1} \times \Phi_{2} \times \Phi_{3}$. Let $x, y \in X$ with $x<y$. In order to verify (2.18), we have to consider the following cases.

Case 1. $x, y \in\left[0, \frac{1}{2}\right]$. It follows that

$$
\begin{aligned}
\phi\left(\int_{0}^{\mathrm{d}(\mathrm{fx}, \mathrm{fy})} \varphi(\mathrm{t}) \mathrm{dt}\right) & =\phi\left(\int_{0}^{\frac{1}{2}|y-x|} \varphi(\mathrm{t}) \mathrm{dt}\right) \\
& =\phi\left(\frac{(y-x)^{2}}{4}\right) \\
& =\frac{(y-x)^{2}}{4} \leqslant(y-x)^{2}-\frac{(y-x)^{2}}{4}=\phi\left(\int_{0}^{\mathrm{d}(x, y)} \varphi(\mathrm{t}) \mathrm{dt}\right)-\int_{0}^{\psi(d(x, y))} \varphi(\mathrm{t}) \mathrm{dt} ;
\end{aligned}
$$

Case 2. $x \in\left(0, \frac{1}{2}\right]$ and $y=1$. Note that

$$
\begin{aligned}
\phi\left(\int_{0}^{\mathrm{d}(f x, f y)} \varphi(t) d t\right) & =\phi\left(\int_{0}^{\frac{x}{2}} \varphi(t) d t\right)=\phi\left(\frac{x^{2}}{4}\right)=\frac{x^{2}}{4} \\
& \leqslant \frac{1}{16}<\frac{3}{16} \leqslant \frac{3}{4}(1-x)^{2}=(1-x)^{2}-\frac{(1-x)^{2}}{4} \\
& =\phi\left(\int_{0}^{d(x, y)} \varphi(t) d t\right)-\int_{0}^{\psi(d(x, y))} \varphi(t) d t
\end{aligned}
$$

Case 3. $x=0$ and $y=1$. It is clear that

$$
\phi\left(\int_{0}^{\mathrm{d}(\mathrm{f} 0, \mathrm{f} 1)} \varphi(\mathrm{t}) \mathrm{dt}\right)=0<1-\frac{1}{16}=\phi\left(\int_{0}^{\mathrm{d}(0,1)} \varphi(\mathrm{t}) \mathrm{dt}\right)-\int_{0}^{\psi(\mathrm{d}(0,1))} \varphi(\mathrm{t}) \mathrm{dt} ;
$$


Case 4. $x \in\left[0, \frac{1}{2}\right]$ and $y=3$. It is clear that

$$
\begin{aligned}
\phi\left(\int_{0}^{\mathrm{d}(\mathrm{fx}, \mathrm{fy})} \varphi(\mathrm{t}) \mathrm{dt}\right)=\phi\left(\int_{0}^{\frac{2-x}{2}} \varphi(\mathrm{t}) \mathrm{dt}\right) & =\phi\left(\left(\frac{2-x}{2}\right)^{2}\right)=\left(\frac{2-x}{2}\right)^{2} \\
& \leqslant 1<2-\frac{x}{2}-\frac{1}{16}=\phi(1+2-x)-\int_{0}^{\frac{1}{4}} \varphi(\mathrm{t}) \mathrm{dt} \\
& =\phi\left(\int_{0}^{1} \varphi(\mathrm{t}) \mathrm{d} \mathrm{t}+\int_{1}^{3-x} \varphi(\mathrm{t}) \mathrm{dt}\right)-\int_{0}^{\psi(3-x)} \varphi(\mathrm{t}) \mathrm{dt} \\
& =\phi\left(\int_{0}^{3-x} \varphi(\mathrm{t}) \mathrm{dt}\right)-\int_{0}^{\psi(3-x)} \varphi(\mathrm{t}) \mathrm{dt} \\
& =\phi\left(\int_{0}^{\mathrm{d}(x, y)} \varphi(\mathrm{t}) \mathrm{dt}\right)-\int_{0}^{\psi(\mathrm{d}(x, y))} \varphi(\mathrm{t}) \mathrm{dt} ;
\end{aligned}
$$

Case 5. $x=1$ and $y=3$. It is easy to verify that

$$
\begin{aligned}
\phi\left(\int_{0}^{\mathrm{d}(\mathrm{fx}, \mathrm{fy})} \varphi(\mathrm{t}) \mathrm{dt}\right)=\phi\left(\int_{0}^{1} \varphi(\mathrm{t}) \mathrm{dt}\right)=\phi(1) & =1 \\
& <\frac{3}{2}-\frac{1}{16} \\
& =\phi(2)-\int_{0}^{\frac{1}{4}} \varphi(\mathrm{t}) \mathrm{dt} \\
& =\phi\left(\int_{0}^{1} \varphi(\mathrm{t}) \mathrm{dt}+\int_{1}^{2} \varphi(\mathrm{t}) \mathrm{dt}\right)-\int_{0}^{\psi(2)} \varphi(\mathrm{t}) \mathrm{dt} \\
& =\phi\left(\int_{0}^{2} \varphi(\mathrm{t}) \mathrm{dt}\right)-\int_{0}^{\psi(2)} \varphi(\mathrm{t}) \mathrm{dt} \\
& =\phi\left(\int_{0}^{\mathrm{d}(x, y)} \varphi(\mathrm{t}) \mathrm{dt}\right)-\int_{0}^{\psi(d(x, y))} \varphi(\mathrm{t}) \mathrm{dt} .
\end{aligned}
$$

That is, (2.18) holds. Thus the conditions of Theorem 2.4 are satisfied. It follows from Theorem 2.4 that $f$ has a unique fixed point in $X$.

\section{Acknowledgment}

This work was supported by the Dong-A University research fund.

\section{References}

[1] M. Abbas, M. A. Khan, Common fixed point theorem of two mappings satisfying a generalized weak contractive condition, Int. J. Math. Math. Sci., 2009 (2009), 9 pages. 1

[2] H. H. Alsulami, E. Karapınar, D. O'Regan, P. Shahi, Fixed points of generalized contractive mappings of integral type, Fixed Point Theory Appl., 2014 (2014), 24 pages. 1

[3] A. Branciari, A fixed point theorem for mappings satisfying a general contractive condition of integral type, Int. J. Math. Math. Sci., 29 (2002), 531-536. 1, 1.3

[4] D. Dey, A. Ganguly, M. Saha, Fixed point theorems for mappings under general contractive condition of integral type, Bull. Math. Anal. Appl., 3 (2011), 27-34. 1

[5] P. N. Dutta, B. S. Choudhury, A generalisation of contraction principle in metric spaces, Fixed Point Theory Appl., 2008 (2008), 8 pages. 1, 1.2

[6] R. George, R. Rajagopalan, Common fixed point results for $\psi-\phi$ contractions in rectangular metric spaces, Bull. Math. Anal. Appl., 5 (2013), 44-52.

[7] V. Gupta, N. Mani, A common fixed point theorem for two weakly compatible mappings satisfying a new contractive condition of integral type, Math. Theory Modeling, 1 (2011), 6 pages. 1 
[8] V. Gupta, N. Mani, Common fixed point for two self-maps satisfying a generalized $\psi \int_{\phi}$ weakly contractive condition of integral type, Int. J. Nonlinear Sci., 16 (2013), 64-71.

[9] V. Gupta, N. Mani, A. K. Tripathi, A fixed point theorem satisfying a generalized weak contractive condition of integral type, Int. J. Math. Anal. (Ruse), 6 (2012), 1883-1889.

[10] V. R. Hosseini, Common fixed point for generalized $(\phi, \psi)$-weak contractions contractions mappings condition of integral type, Int. J. Math. Anal., 4 (2010), 1535-1543.

[11] E. Karapınar, P. Shahi, K. Tas, Generalized $\alpha-\psi$-contractive type mappings of integral type and related fixed point theorems, J. Inequal. Appl., 2014 (2014), 18 pages. 1.6

[12] M. A. Kutbi, M. Imdad, S. Chauhan, W. Sintunavarat, Some integral type fixed point theorems for non-self-mappings satisfying generalized $(\psi, \phi)$-weak contractive conditions in symmetric spaces, Abstr. Appl. Anal., 2014 (2014), 11 pages.

[13] Z.-Q. Liu, J.-L. Li, S. M. Kang, Fixed point theorems of contractive mappings of integral type, Fixed Point Theory Appl., 2013 (2013), 17 pages.

[14] Z.-Q. Liu, X. Li, S. M. Kang, S. Y. Cho, Fixed point theorems for mappings satisfying contractive conditions of integral type and applications, Fixed Point Theory Appl., 2011 (2011), 18 pages. 1

[15] Z.-Q. Liu, H. Wu, J. S. Ume, S. M. Kang, Some fixed point theorems for mappings satisfying contractive conditions of integral type, Fixed Point Theory Appl., 2014 (2014), 14 pages. 1, 1.4, 1.5

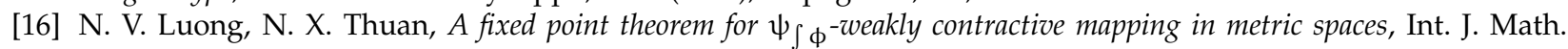
Appl., 4 (2010), 233-242. 1

[17] C. Mongkolkeha, P. Kumam, Fixed point and common fixed point theorems for generalized weak contraction mappings of integral type in modular spaces, Int. J. Math. Math. Sci., 2011 (2011), 12 pages. 1

[18] B. E. Rhoades, Some theorems on weakly contractive maps, Proceedings of the Third World Congress of Nonlinear Analysts, Part 4, Catania, (2000), Nonlinear Anal., 47 (2001), 2683-2693. 1, 1.1

[19] B. E. Rhoades, Two fixed-point theorems for mappings satisfying a general contractive condition of integral type, Int. J. Math. Math. Sci., 2003 (2003), 4007-4013. 1, 1 